\title{
A Gradient-based Optimization Method for Natural Laminar Flow Design
}

\author{
A. Hanifi*, , O. Amoignon*, J. O. Pralits, ${ }^{*,}$, and M. Chevalier*
}

\begin{abstract}
A gradient-based optimization method for minimization of the total drag of an airfoil is presented. The viscous drag is minimized by delaying the laminarturbulent transition. The gradients are obtained solving the adojoint of the Euler, boundary-layer and stability equations. The optimization is subjected to constraints such as restrictions on geometry, lift and pitch moment. The geometry is parametrised using radial basis functions.
\end{abstract}

\section{Introduction}

Drag reduction for high-speed vehicles is a challenging task. In the past, optimization of airfoil mostly aimed at decreasing the pressure drag only neglecting the contribution from viscous drag. However, recent requirements on significant reduction of $\mathrm{CO}_{2}$ and $\mathrm{NO}_{x}$ have resulted in increased interest in laminar airfoil design.

Since laminar-turbulent transition in the boundary-layer flows is usually caused by breakdown of small unstable perturbations, the flow control methods for delay of transition aim at reducing the growth rate of these perturbations. The amplification of boundary-layer disturbances can be analyzed using linear stability theory. The growth rate of the disturbances can then be used to predict the transition location using the so-called $e^{N}$ method, see e.g. van Ingen [12]. Here, it is assumed that transition occurs when the disturbance amplification exceeds an empirically defined threshold. The most common approaches for transition control investigated for the aeronautic applications are wall-suction and shape optimization. The latter is usually denoted as Natural Laminar Flow (NLF) design.

\footnotetext{
*Swedish Defence Research Agency, FOI, SE-164 90 Stockholm, Sweden. e-mail: ardeshir.hanifi@foi.se

${ }^{\dagger}$ Linné Flow Centre, KTH Mechanics, SE-100 44 Stockholm, Sweden

* DIMEC, Università di Salerno, Fisciano (SA), Italy 
CFD-based design optimization has proved to be successful in reducing the pressure drag at transonic flow regime, see e.g. Jameson [7]. However, attempts to minimize the total drag by regular CFD-based design optimization have relied on a fixed laminar-turbulent transition point or on the assumption that the flow is fully turbulent as in Nemec \& Zingg [9]. Linear stability analysis has been used in a number of investigations for NLF design. In Green \& Whitesides [5], a target pressure is found, based on a simplified relation between pressure and disturbance amplification (N-factor), which is used to state an inverse problem in order to find a geometry that may delay transition. Streit \& Liersch [11] also used an inverse method to design a transonic wing with natural laminar flow. In Manning \& Kroo [8], a surface panel method was coupled with an approximative boundary layer calculation and linear stability analysis. Iuliano et al. [6] used a genetic algorithm for NLF design of a supersonic transport jet wing-body. However, none of these investigations calculated the sensitivities based on the linear stability analysis in order to formulate optimality conditions.

The method for NLF design presented here is gradient-based and utilizes the adjoints of the Euler, boundary-layer and stability equations to calculate the required gradients. Here, CFD analyses are coupled to boundary-layer stability computations. With this approach, the geometry, here an airfoil, can be optimized with respect to the disturbance amplification in order to delay the laminar-turbulent transition.

\section{Governing equations}

The most common transition prediction methods are based on the amplification rate of harmonic disturbances imposed to the boundary layer. Once the boundary-layer profiles are given the convective instability of these disturbances can be obtained with good accuracy using, for example, the nonlocal stability theory solving the Parabolized Stability Equations (PSE), see e.g. Bertolotti et al. [4]. The amplification rates of perturbations are known to be sensitive to accuracy of the computed profiles. Usually, the required accuracy is not achieved through Navier-Stokes (NS) computations due to the high resolution needed. Therefore, often the profiles are obtaind as solutions of the boundary-layer equations performed using pressure distribution given by Euler or Navier-Stokes computations as input. This approach has been used here. The governing equations in symbolic form can be written as

$$
\begin{aligned}
\mathscr{L}_{e}(\mathbf{w}, \Gamma) & =\mathbf{0}, \\
\mathscr{L}_{\text {ble }}\left(\mathbf{Q}, \mathbf{w}, \Gamma_{\text {ble }}\right) & =\mathbf{0}, \\
\mathscr{L}_{\text {pse }}\left(\mathbf{q}, \mathbf{Q}, \Gamma_{\text {pse }}\right) & =\mathbf{0} .
\end{aligned}
$$

Here, $\mathscr{L}_{e}, \mathscr{L}_{\text {ble }}$ and $\mathscr{L}_{\text {pse }}$ represent operators corresponding to Euler, boundarylayer and disturbance equations, respectively. Further, w denotes the inviscid flow field, $\mathbf{Q}$ the viscous boundary-layer profiles and $\mathbf{q}$ the perturbation quantities. The 
whole geometry is notated by $\Gamma$ while,$\Gamma_{b l e}$ and $\Gamma_{p s e}$ represent the part of geometry considered for boundary-layer and stability computations, respectively.

The viscous-inviscid interaction is neglected here. In this way the Euler equations $\mathscr{L}_{e}$ only depend on the shape $\Gamma$ at given flow conditions (angle of attach, Mach and Reynolds numbers). The inviscid flow $\mathbf{w}$ on $\Gamma_{\text {ble }}$ provides the boundary conditions for the boundary-layer equations $\left(\mathscr{L}_{\text {ble }}\right)$. The flow in the laminar boundary layer $\mathbf{Q}$ and geometry $\Gamma_{p s e}$ define the coefficients of the PSE, for given flow conditions and for chosen disturbance parameters (frequency and wave number).

\section{Optimization problem}

As mentioned above the laminar flow control problem and the aerodynamic shape optimization can be defined as an optimization problem which mathematically can be formulated as:

$$
\min _{\mathbf{a} \in \mathbf{R}^{n} d} J(\mathbf{q}, \mathbf{w}, \Gamma) \quad \text { subject to }\left\{\begin{aligned}
f_{j}(\mathbf{q}, \mathbf{w}, \Gamma) \leq 0,1 \leq j \leq m, \\
\mathscr{L}_{e}(\mathbf{w}, \Gamma)=\mathbf{0}, \\
\mathscr{L}_{\text {ble }}\left(\mathbf{Q}, \mathbf{w}, \Gamma_{b l e}\right)=\mathbf{0}, \\
\mathscr{L}_{p s e}\left(\mathbf{q}, \mathbf{Q}, \Gamma_{p s e}\right)=\mathbf{0}, \\
\mathscr{S}(\Gamma, \mathbf{a})=\mathbf{0},
\end{aligned}\right.
$$

where $J$ is a cost function, $f_{j}$ are the constraints. The cost function $J$ and the constraints $f_{j}$ are explicit functionals of one or several of the variables $\{\mathbf{q}, \mathbf{w}, \Gamma\}$. Further, $\mathscr{S}$ denotes the parameterization of the geometry which defines the geometry $\Gamma$ for given control variables a.

Since we aim to minimize the pressure drag as well as the friction drag the considered objective function should include measures of both of them. The friction drag is thought to be minimized by delaying the laminar-turbulent transition. This is addressed by reducing the amplification of boundary-layer perturbations as much as possible through variation of geometry (pressure gradient). Usually, different types of disturbances (e.g. cross-flow vortices and Tollmien-Schlichting waves) are present in the boundary layer simultaneously. These disturbances react differently to changes in pressure distribution. Further, perturbations of the same type are dominating at different streamwise positions. Therefore, the measure of disturbance amplitude should include contribution of different perturbations.

The measure of disturbances used here is based on the kinetic energy of a number of perturbations integrated over a defined domain

$$
E=\frac{1}{2} \sum_{k=1}^{K} \int_{\Omega_{p s e}} \mathbf{q}_{k}^{H} \mathbf{M q}_{k} d \Omega,
$$

where $\mathrm{M}$ is a weighting matrix. This measure has successfully been used in optimization of wall-suction distribution for transition control, see Pralits \& Hanifi[10] . Then, the objective function $J$ is defined as 


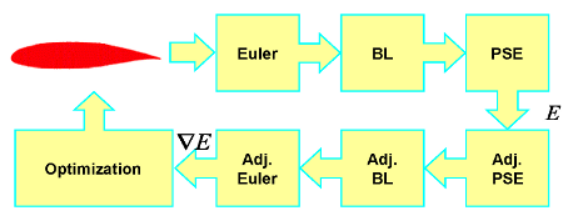

Fig. 1 Schematic of optimization procedure.

$$
J=\lambda_{U} E+\lambda_{D} C_{D},
$$

where $C_{D}$ is the inviscid pressure drag. Here, $\lambda_{U}$ and $\lambda_{D}$ are weight parameters. The optimizations can also be constrained by requirements on values of lift and pitch moment. Constraints are further imposed on the geometry features as constant cross-sectional area, fixed trailing edge, and a fixed region of the airfoil around the leading edge. These constraints can be treated as equality or inequality constraints like minimum thickness and minimum lift. As mentioned before the optimization alghorithms used here are gradient-based. The gradients are efficiently obtained solving adjoints of the Euler, boundary-layer and disturbance equations. The gradient of the disturbance energy is computed using the chain rule

$$
\frac{\partial E}{\partial \mathbf{a}}=\frac{\partial E}{\partial \mathbf{Q}} \times \frac{\partial \mathbf{Q}}{\partial \mathbf{w}} \times \frac{\partial \mathbf{w}}{\partial \mathbf{a}} .
$$

The gradients in the right hand side of the expression above represent solutions of the adjoint equations. This expression is equivalent to the following chain of computations

$$
\underbrace{\mathrm{APSE} \rightarrow \mathrm{ABLE}}_{\text {cont. }} \rightarrow \underbrace{\text { adjoint Euler. }}_{\text {discr. }}
$$

Adjoint of the stability equations (APSE) and boundary-layer equations (ABLE) are derived in contineous form and then discretised while adjoint of the Euler equations are derived directly from the discretised ones. Details of derivation and implementation of these equations can be found in references $[3,2]$. The schematic of the optimization procedure is given in Fig. 1.

\section{Results}

Here, results of the optimization of two-dimensional airfoils are presented. The aim is to demonsterate the behavior of our method. The geometries correspond to the tip and root sections of a low speed aircraft at a free-stream Mach number of $M_{\infty}=$ 0.374. The initial geometry is a NASA TP 1786 airfoil with 17\% thickness. The optimization problem is formulated as: 

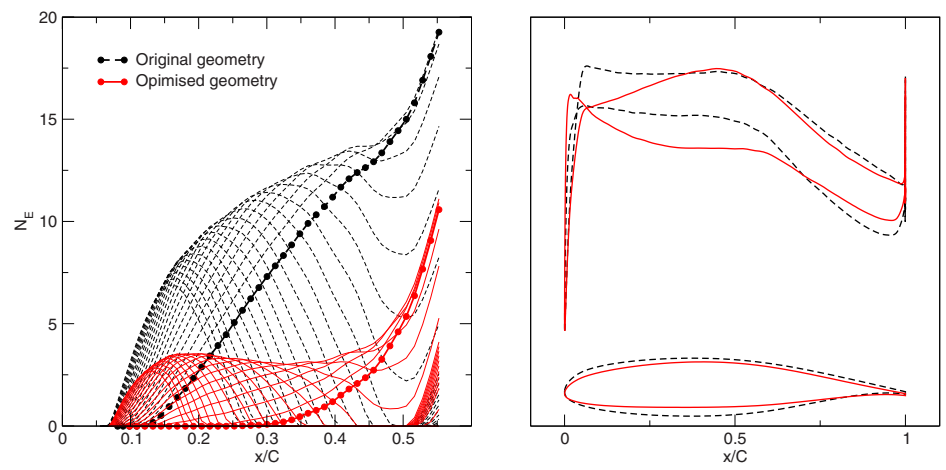

Fig. 2 NLF airfoil optimization at $M_{\infty}=0.374$ and $R e=7 \times 10^{6}$ corresponding to the tip section. Right: Pressure coefficients $-C_{p}$ and unscaled geometry. Left: $N_{E}$-factor curves. The dashed lines denote initial design and the solid lines the final design. Symbols denotethe target disturbane.

$$
\min _{\Gamma} \log (E)+0.1 \frac{C_{D}}{C_{D}{ }^{0}} \quad \text { subject to } \quad C_{L} \geq C_{L}{ }^{0}, C_{m} \geq C_{m}{ }^{0}, t \geq t^{\min }
$$

where the superscript ${ }^{0}$ indicates values at the initial design (baseline), $t$ the airfoil thickness and superscript $\min$ refers to the minimum avalue allowed. Further, as the value of the disturbance energy $E$ varies a lot in the course of optimization the $\log (E)$ is used instead. Here, the Radial Basis Functions (RBF) have been used to parametrize the geometry. The details of its implementation can be found in reference [1].

In Fig. 2 results of optimization of the airfoil corresponding to the tip section of the wing are presented. Here, the minimum thickness is $t^{\mathrm{min}} / C=12 \%$ and the Reynolds number based on the chord length $C$ is $R e=7 \times 10^{6}$ and $C_{L}^{0}=0.3$. The optimization aimed at reducing the disturbance growth on the upper side of the airfoil only. The targeted disturbance is a two-dimensional Tollmien-Schlichting (TS) wave at $5 \mathrm{kHz}$ (marked with symbols). The $N_{E}$-factor curves plotted in Fig. 2 are defined as

$$
N_{E}(x)=0.5 \cdot \ln \left(\frac{\hat{E}(x)}{\hat{E}\left(x_{0}\right)}\right),
$$

where $\hat{E}$ is the kinetik energy of disturbances and $x_{0}$ refers to the location the disturbance energy first starts to grow. Here, the critical value of $N_{E}$-factor is assumed to be around 10. As can be seen in Fig. 2, the disturbance amplification is reduced significantly and its value is well below the citical one every where. The boundary layer computations stop around $x / C \approx 0.55$. The final geometry has a an accelerating flow in a large area of the upper side. This is known to have a damoing effect on the growth of TS-waves. Plots in Fig. 3 show the history of the computations corresponding to the results presented in Fig. 2.

In Fig. 4 results of optimization of an airfoil corresponding to the root section of the wing are presented. Here, the minimum thickness is $t^{\text {min }} / C=16 \%$ and the 

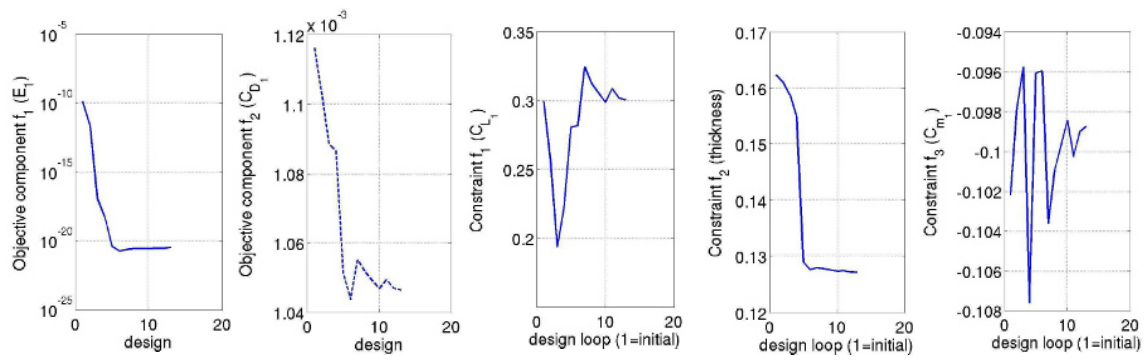

Fig. 3 Optimization history corresponding to the results of Fig. 2.

Reynolds number based on the chord length $R e=12.1 \times 10^{6}$ and $C_{L}^{0}=0.4$. As for the tip section, the energy of a two-dimensional TS wave at $5 \mathrm{kHz}$ on the upper surface of the wing is to be reduced. Besides the results for final geometry, results for an intermediate one are also plotted there. The pressure distribution on both sides are similar to that on the optimized tip section. However, here the pressure gradient on the upper side is stronger resulting in a more pronounced stabilisation of disturbances. As mentioned above, the optimization is based on the inviscid computations. In order to analyse the correct behaviour of the optimized airfoil, RANS computations with prescribed transition point were performed. The location of transition was predicted as the streamwise position where the envelope of the $N$-factor curves reached a value of 10 . Results of these computations, in terms of $C_{L}$ and $C_{D}$, are given in Fig. 5. Here, $C_{D}$ includes both the pressure and viscous drag. As can be observed there, for low and moderate lift coefficients the performance of the optimized airfoil is better than the initial geometry. However, the optimized airfoil loses lift at higher angle of attack. This is due to the fact that at these angle of attacks boundary-layer separation occurs earlier on the optimized airfoil than on the initial one. This clearly shows the need for inclusion of the viscous effects in the optimization procedure.

In the previous examples only the upper side of the airfoil has been considered for laminar flow optimization. The resulted lower surface pressure distributions have stronger adverse gradients than that from the original geometries, resulting in earlier transition on the lower side of the optimized geometries than on the original ones. Therefore, we examined the possibility to extend the laminar flow on both side of the airfoil. Results of the computations are given in Fig. 6. Here, the objective function includes the contributions from disturbances on both side of the airfoil. As can be seen there, the disturbances are highly damped on both upper and lower surfaces of the optimized airfoil. 

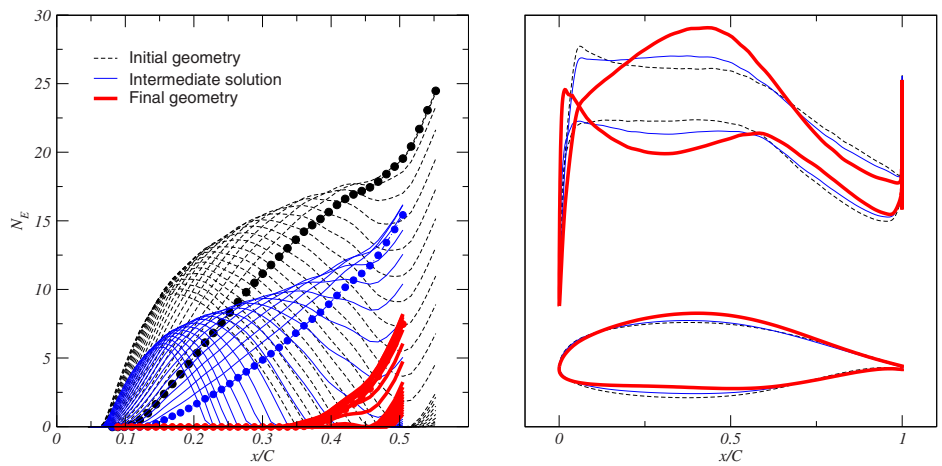

Fig. 4 NLF airfoil optimization at $M_{\infty}=0.374$ and $R e=12.1 \times 10^{6}$ corresponding to the root section. Upper side optimized for NLF. Right: Pressure coefficients $-C_{p}$ and unscaled geometry. Left: $N_{E}$-factor curves. The dashed lines denote initial design and the solid lines the intermediate and final design (thick line). Symbols denote the target disturbane

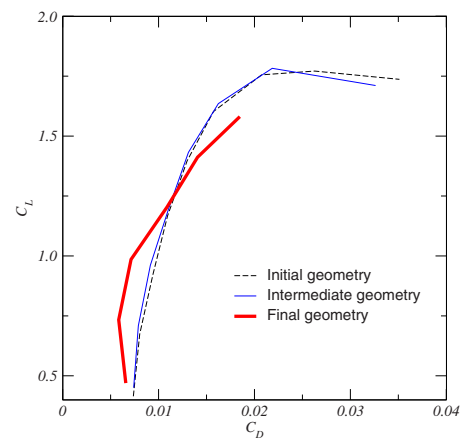

Fig. 5 Lift and drag coeeficients for airfoil given in Fig. 6. RANS computations with transition point given by stability analyses.

\section{Conclusions}

Utilizing the adjoint equations, the gradients of amplification of the boundarylayer disturbances with respect to geometry variations have been efficiently computed. The method couples the Euler, boundary-layer and stability equations as well as their adjoints to compute the gradients. The results of optimization of twodimensional airfoils have been presented indicating that the method is able to produce NLF airfoils. Further, RANS computations on the optimized geometries with prescribed transition location showed that inclusion of viscous effects in optimization procedure may be necessary in order to account for the effects of flow separation. 

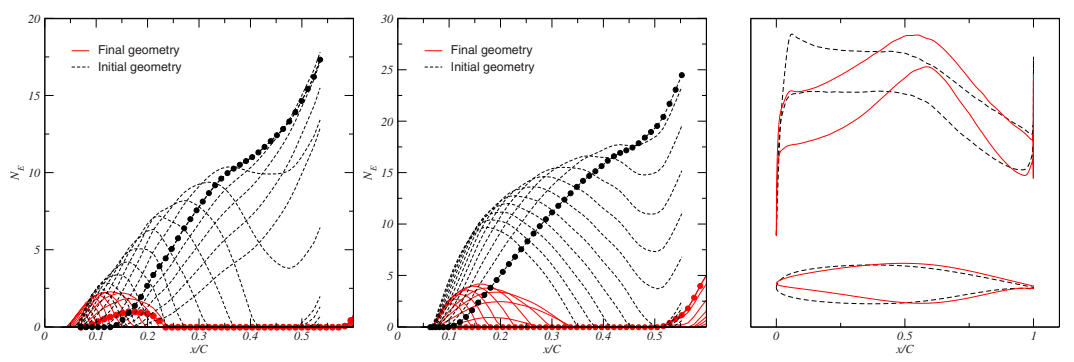

Fig. 6 NLF airfoil optimization at $M_{\infty}=0.374$ and $R e=12.1 \times 10^{6}$ corresponding to the root section. Both upper and lower side are optimized for NLF. Right: Pressure coefficients $-C_{p}$ and unscaled geometry. Middle: $N_{E}$-factors curves on the upper side. Left: $N_{E}$-factor curves on the lower side. The dashed lines denote initial design and the solid lines the final design. Symbols denote the target mode.

Acknowledgements Parts of this work have been funded by European Commission through SUPERTRAC project (contract no: AST4-CT-2005-516100) and CESAR project (contract no: AIP5CT-2006-030888).

\section{References}

1. O. Amoignon. AESOP - A Numerical Platform for Aerodynamic Shape Optimization. $J$. Optimization and Engineering, DOI 10.1007/s11081-008-9078-7, 2008.

2. O. Amoignon and M. Berggren. Adjoint of a median-dual finite-volume scheme: Application to transonic aerodynamic shape optimization. Technical Report 2006-013, Department of Information Technology, Uppsala University, Uppsala, Sweden, 2006.

3. O. Amoignon, J. O. Pralits, A. Hanifi, M. Berggren, and D. S. Henningson. Shape optimization for delay of laminar-turbulent transition. AIAA J., 44(5):1009-1024, 2006.

4. F. P. Bertolotti, Th. Herbert, and S.P. Spalart. Linear and nonlinear stability of the Blasius boundary layer. J. Fluid Mech., 242:441-474, 1992.

5. B. E. Green and J. L. Whitesides. A method for the constrained design of natural laminar flow airfoils. AIAA Paper, (96-2502), 1996.

6. E. Iuliano, R. Donelli, D. Quagliarella, I. Salah El Din, and D. Arnal. Natural laminar flow design of a supersonic transport jet wing-body. AIAA paper 2009-1279, 2009.

7. A. Jameson. Optimum aerodynamic design using CFD and control theory. AIAA Paper, (951729), 1995.

8. V. M. Manning and I. M. Kroo. Multidisciplinary optimization of a natural laminar flow supersonic aircraft. AIAA Paper, (99-3102), 1999.

9. M. Nemec and D.W. Zingg. Towards efficient aerodynamic shape optimization based on the Navier-Stokes equations. AIAA Paper, (2001-2532), 2001.

10. J. O. Pralits and A. Hanifi. Optimization of steady suction for disturbance control on infinite swept wings. Phys. Fluids, 15(9):2756-2772, 2003.

11. T. Streit and C. Liersch. Design of a transonic wing with natural laminar flow for the EC project NACRE. KATnet II Drag Reduction Workshop. Oct. 14-16, 2008. Ascot, UK., 2008.

12. J. L. van Ingen. A suggested semiempirical method for the calculation of the boundary layer transition region. Technical Report VTH-74, Department of Aeronautical Engineering, University of Delft, 1956. 